But it shows at any rate that any effort to find a contradiction between $\gamma, \delta, \epsilon$, or any combination of these, would be futile.

We have failed to find a map on which all of the operations of the impasse group are possible.

NeW YORK UNIVERSITY

\title{
ON THE EQUIVALENCE OF TWO METHODS OF DEFINING STIELTJES INTEGRALS*
}

BY B. C. GETCHELL

1. Introduction. The Stieltjes integral, $\int_{a}^{b} \phi(x) d g(x)$, was originally defined for $\phi(x)$ continuous on the closed interval $[a, b]$, and $g(x)$ of bounded variation. The limit which gives rise to this integral is taken as the length of the greatest sub-interval approaches zero. The above restrictions on $\phi(x)$ and $g(x)$, however, are not at all necessary for the existence of the limit, although it fails when the two functions have a common point of discontinuity. A generalization which permits such discontinuities is obtained by taking the limit in the sense of subdivisions, $\dagger$ to be defined below. The Riemann integral is an instance of the first type of limiting process, while the associated Darboux integrals are of the subdivision type. These can be shown to be of the first type as well. It is the purpose of this note to obtain general conditions for the equivalence of the two limits. By the introduction of the notion of interval functions a simple restriction on the integrand is found to be both necessary and sufficient.

2. Subdivisions. By a subdivision, $\sigma$, of the linear interval $X=[a, b]$ will be understood a finite set of adjacent sub-intervals whose sum is $X$. The norm of $\sigma$, the length of the greatest sub-interval, will be written $N_{\sigma}$. By the product, $\sigma^{\prime} \cdot \sigma^{\prime \prime}$, of two subdivisions of $X$ will be understood the subdivision which consists of all products of the form $I^{\prime} \cdot I^{\prime \prime}$, where $I^{\prime}$ is an element of $\sigma^{\prime}$, and $I^{\prime \prime}$ is an element of $\sigma^{\prime \prime}$. It is assumed that every such

* Presented to the Society, April 6, 1934.

$\dagger$ Moore and Smith, $A$ general theory of limits, American Journal of Mathematics, vol. 44 (1922), pp. 102-121. 
pair of subdivisions has a product. Whenever two subdivisions $\sigma$ and $\sigma^{\prime}$ of $X$ satisfy the condition $\sigma=\sigma \cdot \sigma^{\prime}$ we shall write $\sigma \geqq \sigma^{\prime}$. This is equivalent to having every element of $\sigma$ contained in or identical with some element of $\sigma^{\prime}$.

3. Interval Functions. An interval function, $f(I)$, on $X$ will be any relation which assigns one or more finite real values to each sub-interval $I$ which is an element of a subdivision of $X$. The expression $f(I)=\phi(\xi)\left[g\left(x^{\prime \prime}\right)-g\left(x^{\prime}\right)\right], x^{\prime} \leqq \xi \leqq x^{\prime \prime}$, where $\phi(x)$ and $g(x)$ are bounded point functions, and $x^{\prime}, x^{\prime \prime}$ are the left and right end points of $I$, may be taken as an example.

4. Integrals. The symbol $\sum_{\sigma} f(I)$ will be used to designate any sum of the values of $f(I)$ obtained by choosing one value to correspond to each element of $\sigma$. It is a function of $\sigma$ and may be multiple valued. The norm and sigma integrals of $f(I)$ on $X$, written $N \int_{X} f(I)$ and $\sigma \int_{X} f(I)$, respectively, will be the corresponding limits of $\sum_{\sigma} f(I)$ with respect to the subdivisions of $X$. These limits are defined explicitly as follows.

(1) The norm limit. If there exists a finite number $A$ such that for every positive number $e$ a positive $N_{e}$ can be found which makes* $L U B\left|\sum_{\sigma} f(I)-A\right| \leqq e$ whenever $\sigma$ is a subdivision of $X$ with $N_{\sigma} \leqq N_{e}$, we write $\lim _{N} \sum_{\sigma} f(I)=A$.

(2) The sigma limit. If there exists a finite number $A$ such that for every positive $e$ there can be found a subdivision $\sigma_{e}$ of $X$ with respect to which $L U B\left|\sum_{\sigma} f(I)-A\right| \leqq e$ whenever $\sigma \geqq \sigma_{\epsilon}$, we write $\lim _{\sigma} \sum_{\sigma} f(I)=A$. This is the limit in the sense of subdivisions mentioned above. It is easily shown to exist and to be equal to the norm limit when the latter exists.

5. Additive and Pseudo-Additive Functions. Let $I^{\prime}$ and $I^{\prime \prime}$ be a pair of adjacent intervals with the common end point $x$. Consider now the expression

$$
\Delta(I, x)=L U B\left|f\left(I^{\prime}\right)+f\left(I^{\prime \prime}\right)-f(I)\right|,
$$

where $I=I^{\prime}+I^{\prime \prime}$. The function $f(I)$ is said to be additive if $\Delta(I, x)$ is zero for every such pair. We shall need a weaker condition. If $\lim \Delta(I, x)=0$ as the length of $I$ goes to zero, we shall say that $f(I)$ is pseudo-additive at $x$. The function will be called pseudo-additive on the interval $X$ if it has the property at every

* $L U B=$ least upper bound. 
point. When elements of zero length (points) are permitted, it becomes possible for three sub-intervals to be adjacent in the sense that they have a common end point. Then $\Delta(I, x)$ must take the form

$$
L U B\left|f\left(I^{\prime}\right)+f\left(I^{\prime \prime}\right)+f\left(I^{\prime \prime \prime}\right)-f(I)\right| .
$$

The continuous interval functions are necessarily pseudo-additive.

6. Condition for Equivalence of Integrals. As stated in the introduction, the sigma integral may exist when the corresponding norm integral fails. The following theorem gives the condition for their equivalence.

Theorem. For the existence of $N \int_{X} f(I)$ it is necessary and sufficient that $\sigma \int_{X} f(I)$ exist and that $f(I)$ be pseudo-additive on $X$.

Necessity. Since the existence of the norm integral implies that of the sigma integral, it must be shown only that $f(I)$ is pseudoadditive on $X$ whenever the former integral exists. If the contrary is assumed, there must be a point $x$ of $X$ and a positive number $e_{0}$ such that for every $N>0$ there can be found a pair of adjacent intervals $I^{\prime}$ and $I^{\prime \prime}$ of $X$ with the following properties: $I^{\prime}$ and $I^{\prime \prime}$ have $x$ as a common end point, their sum, $I$, has a length not greater than $N$, and $L U B\left|f\left(I^{\prime}\right)+f\left(I^{\prime \prime}\right)-f(I)\right|>e_{0}$. Now choose a subdivision $\sigma^{\prime}$ of $X$ with $I^{\prime}$ and $I^{\prime \prime}$ as elements and with norm less than or equal to $N$. Let $\sigma^{\prime \prime}$ be the subdivision obtained by the substitution of $I$ for its components in $\sigma^{\prime}$. Then

$$
L U B\left|\sum_{\sigma^{\prime}} f(I)-\sum_{\sigma^{\prime \prime}} f(I)\right| \geqq L U B\left|f\left(I^{\prime}\right)+f\left(I^{\prime \prime}\right)-f(I)\right|>e_{0} .
$$

Since $N$ is arbitrary, the hypothesis that $N \int_{X} f(I)$ exists cannot hold.

Sufficiency. From the definition of the sigma integral it follows that for every positive $e$ there exists a subdivision $\sigma_{e}$ of $X$ such that

$$
L U B\left|\sum_{\sigma} f(I)-\sigma \int_{X} f(I)\right| \leqq e / 2
$$

whenever $\sigma \geqq \sigma_{\theta}$. Let $x_{1}, \cdots, x_{n}$ be the end points of the elements of $\sigma_{e}$. If $k$ is the number of its sub-intervals, it is possible to find, because $f(I)$ is pseudo-additive, an $N_{\theta}^{\prime}>0$ such that 


$$
L U B\left|f\left(I^{\prime}\right)+f\left(I^{\prime \prime}\right)-f(I)\right| \leqq e /(2 k)
$$

whenever the length of $I$ is not greater than $N_{e}^{\prime}$, and one of the points $x_{1}, \cdots, x_{n}$ is a common end point of $I^{\prime}$ and $I^{\prime \prime}$. If $N_{\theta}^{\prime \prime}$ is one-half the least distance between any two of the above points, let the lesser of $N_{e}^{\prime}$ and $N_{e}^{\prime \prime}$ be designated by $N_{e}$. Then, by reason of (2), it follows that

$$
L U B\left|\sum_{\sigma^{\prime}} f(I)-\sum_{\sigma} f(I)\right| \leqq k e /(2 k)=e / 2
$$

whenever $N_{\sigma^{\prime}} \leqq N_{e}$ and $\sigma=\sigma^{\prime} \cdot \sigma_{e} \geqq \sigma_{e}$. Therefore

$$
L U B\left|\sum_{\sigma^{\prime}} f(I)-\sigma \int_{X} f(I)\right| \leqq e
$$

by reason of (1) and (3), and the norm integral exists. It is assumed that the points $x_{1}, \cdots, x_{n}$ are all distinct, that is, that sub-intervals of zero length are excluded. The proof is easily modified to allow for this case also.*

Example 1. The elements of subdivisions in the first four examples will be closed intervals, and $f(I)=\left|g\left(x^{\prime \prime}\right)-g\left(x^{\prime}\right)\right|$, where $x^{\prime}$ and $x^{\prime \prime}$ are the left and right end points of $I$. If $g(x)$ is of bounded variation, then $\sigma \int_{X} f(I)$ exists and is equal to the total variation of $g(x)$ on $X$. The theorem just proved gives the well known condition

$$
|g(x)-g(x-0)|+|g(x+0)-g(x)|=|g(x+0)-g(x-0)|
$$

at each inner point of $X$ for the existence of the total variation as a norm integral.

EXAMPLE 2. $f(I)=\phi(\xi)\left[g\left(x^{\prime \prime}\right)-g\left(x^{\prime}\right)\right], x^{\prime} \leqq \xi \leqq x^{\prime \prime} ; \phi(x)$ and $g(x)$ are bounded point functions. In this case $\Delta(I, x)$ takes the form

$$
\begin{aligned}
& L U B \mid\left[\phi\left(\xi^{\prime}\right)-\phi(\xi)\right]\left[g(x)-g\left(x^{\prime}\right)\right] \\
& \quad+\left[\phi\left(\xi^{\prime \prime}\right)-\phi(\xi)\right]\left[g\left(x^{\prime \prime}\right)-g(x)\right] \mid
\end{aligned}
$$

* After this note had been sent to press, T. H. Hildebrandt called the author's attention to an article by Gustav Doetsch, Journal für Mathematik, August, 1934, in which the property referred to here as pseudo-additive is shown to be sufficient for the existence of the norm integral in the special case of monotonic interval functions. Application is made to extensions of the Darboux theorem on upper and lower integrals. 
$x^{\prime} \leqq \xi^{\prime} \leqq x \leqq \xi^{\prime \prime} \leqq x^{\prime \prime} . \operatorname{Lim} \Delta=0$ at any point where $\phi(x)$ or $g(x)$ is continuous. To show that the converse is also true we assume that they have a common point of discontinuity at $x$. Then there exists an $e_{0}>0$ such that for every positive number $N$ two points $x_{1}$ and $x_{2}$ can be found which satisfy the inequalities $\left|x_{2}-x\right|$ $\leqq\left|x_{1}-x\right| \leqq N / 2,\left|g\left(x_{1}\right)-g(x)\right|>\sqrt{ } e_{0}$, and $\left|\phi\left(x_{2}\right)-\phi(x)\right|>\sqrt{ } e_{0}$. Without loss of generality it may be assumed that $x_{1}<x$. In the above expression for $\Delta$ let $x^{\prime}=x_{1}$ and $x^{\prime \prime}=x+N / 2$. If $x_{2}<x$ let $\xi=\xi^{\prime \prime}=x$ and $\xi^{\prime}=x_{2}$. On the other hand, if $x<x_{2}$ take $\xi=\xi^{\prime \prime}=x_{2}$ and $\xi^{\prime}=x$. In either case

$$
\Delta(I, x) \geqq\left|\left[\phi\left(x_{2}\right)-\phi(x)\right]\left[g(x)-g\left(x_{1}\right)\right]\right|>\left(\sqrt{ } e_{0}\right)^{2}=e_{0} .
$$

But $\left|x^{\prime \prime}-x^{\prime}\right| \leqq N$, an arbitrary number. It follows that $f(I)$ is pseudo-additive on $X$ if and only if $\phi(x)$ and $g(x)$ have no common point of discontinuity.

Example 3. Substitute $x^{\prime}<\xi<x^{\prime \prime}$ for $x^{\prime} \leqq \xi \leqq x^{\prime \prime}$ in Example 2 . The resulting integrals* are more general but reduce to the corresponding integrals of the earlier example whenever these exist. The condition that $f(I)$ be pseudo-additive is the same, with the exception that $\phi(x)$ and $g(x)$ may have removable discontinuities at the same point. If either discontinuity is not of this type, the norm integral fails to exist, unless the point in question is an end point of $X$. Consider the case $\phi(x)=g(x)=0, x \neq x_{0}$; $\phi\left(x_{0}\right)=g\left(x_{0}\right)=1, x_{0}$ an inner point of $X$. Both integrals exist and have the value zero. Under the definition of $f(I)$ in the previous example neither integral exists.

Example 4. $f(I)=\left[\phi\left(x^{\prime \prime}\right)+\phi\left(x^{\prime}\right)\right]\left[g\left(x^{\prime \prime}\right)-g\left(x^{\prime}\right)\right] / 2 . \dagger$

$$
2 \Delta(I, x)=\left|\begin{array}{lll}
g(x) & \phi(x) & 1 \\
g\left(x^{\prime}\right) & \phi\left(x^{\prime}\right) & 1 \\
g\left(x^{\prime \prime}\right) & \phi\left(x^{\prime \prime}\right) & 1
\end{array}\right| .
$$

If the sigma integral exists, the vanishing of the determinant at each inner point of $X$ as $x^{\prime}$ and $x^{\prime \prime}$ approach $x$ independently from opposite sides is a necessary and sufficient condition for the existence of the norm integral.

* B. Dushnik, Dissertation, University of Michigan, 1931, p. 13.

$\dagger$ H. L. Smith, Transactions of this Society, vol. 27 (1925), p. 491. See also H. S. Kaltenborn, Dissertation, University of Michigan, 1934. 
Example 5. Subdivisions consist of open intervals and points. $f(I)=\phi(\xi)\left[g\left(x^{\prime \prime}-0\right)-g\left(x^{\prime}+0\right)\right], x^{\prime}<\xi<x^{\prime \prime}$, if $I$ is an interval. For the point $x, f(I)=\phi(x)[g(x+0)-g(x-0)] .{ }^{*}$ It is assumed that both functions are bounded and that $g(x)$ has no discontinuities of the second kind. In view of the remark concerning subdivisions with point elements we obtain an expression for $\Delta(I, x)$ which may be written as the least upper bound of

$$
\begin{aligned}
& \mid[\phi(x)-\phi(\xi)][g(x+0)-g(x-0)] \\
& \quad+\left[\phi\left(\xi^{\prime}\right)-\phi(\xi)\right]\left[g(x-0)-g\left(x^{\prime}+0\right)\right] \\
& \quad+\left[\phi\left(\xi^{\prime \prime}\right)-\phi(\xi)\right]\left[g\left(x^{\prime \prime}-0\right)-g(x+0)\right] \mid .
\end{aligned}
$$

The test in this case is the behavior of the first term; the remainder of the expression always goes to zero with the length of $I$. Therefore $\lim \Delta=0$ if and only if $\phi(x)$ is continuous or $g(x)$ has at most a removable discontinuity at $x$.

If $g(a)$ and $g(b)$ are substituted for $g(a-0)$ and $g(b+0)$ respectively in the above then $N \int_{X} f(I)$ reduces to the Young integral $\dagger \int_{a}^{b} \phi(x) d g(x)$ obtained by integrating

$$
\begin{aligned}
F(I)=\phi(\xi)\left[g\left(x^{\prime \prime}-0\right)-g\left(x^{\prime}+0\right)\right] & +\phi\left(x^{\prime}\right)\left[g\left(x^{\prime}+0\right)-g\left(x^{\prime}\right)\right] \\
& +\phi\left(x^{\prime \prime}\right)\left[g\left(x^{\prime \prime}\right)-g\left(x^{\prime \prime}-0\right)\right],
\end{aligned}
$$

$x^{\prime}<\xi<x^{\prime \prime}$, over the closed interval $X=[a, b]$, with subdivisions consisting of closed intervals as in the previous examples. The conditions for the existence of the Young integral are the same as those at the end of the preceding paragraph.

Needham, Mass.

* A. Kolmogoroff, Untersuchungen über den Integralbegriff, Mathematische Annalen, vol. 103 (1930), pp. 654-696.

$\dagger \mathrm{W}$. $\mathrm{H}$. Young, On integration with respect to a function of bounded variation, Proceedings of the London Mathematical Society, (2), vol. 13 (1913), p. 113. 